\title{
Bispectral Index Monitoring Reduces the Dosage of Propofol and Adverse Events in Sedation for Endobronchial Ultrasound
}

\author{
Natividad Quesada ${ }^{a}$ Diego Júdez ${ }^{c} \quad$ Javier Martínez Ubieto ${ }^{a} \quad$ Ana Pascuala $^{a}$ \\ Enrique Chacón ${ }^{b}$ Francisco De Pablo ${ }^{b}$ Elisa Mincholé ${ }^{b}$ Salvador Bello ${ }^{b}$ \\ Servicios de a Anestesiología, Reanimación y Terapéutica del Dolor and beumología, Hospital Universitario \\ Miguel Servet, Zaragoza, and 'Servicio de Anestesiología, Reanimación y Terapéutica del Dolor, Hospital de Alcañiz, \\ Teruel, Spain
}

\section{Key Words}

Adverse drug reactions $\cdot$ Anesthesia $\cdot$ Endobronchial ultrasound $\cdot$ Bispectral index

\begin{abstract}
Background: Current guidelines recommend monitoring the anesthetic depth of sedation during respiratory endoscopy by using clinical scales despite their subjective nature and the potential change in the level of sedation caused by frequent stimulation. Monitoring by means of the bispectral index (BIS) has shown its utility in reducing the use of drugs and their adverse events in general anesthesia, but evidence in prolonged sedation is insufficient. Objective: Our objective was to evaluate BIS in patients undergoing endobronchial ultrasound (EBUS). Methods: A randomized cohort study of 90 patients with mediastinal lymph node involvement and/or lung or mediastinal lesions for whom EBUS was indicated, comparing the modified observer's assessment of alertness/sedation scale clinical evaluation $(n=45)$ versus the BIS evaluation $(n=45)$ of sedation with propofol-remifentanil, was conducted in order to evaluate the clinical parameters, doses used, adverse events, and tolerance of the procedure. Results: We found a shorter waking time and a significantly lower dose of total propofol in the BIS group.
\end{abstract}

Significantly fewer overall adverse events were recorded in the BIS group and included desaturation, hypotension, and bradypnea. Tolerance was better in the BIS group. No significant differences were found in terms of cough, memory of the procedure, or the level of difficulty of EBUS on the part of the pulmonologists. Conclusions: BIS monitoring of sedation in EBUS makes it possible to reduce the dosage of propofol, thereby shortening the waking time and reducing adverse events. This form of monitoring should be taken into consideration in the future for systematic use in prolonged sedation, as in the case of EBUS.

(c) 2016 S. Karger AG, Basel

\section{Introduction}

Endobronchial ultrasound-guided transbronchial needle aspiration (EBUS-TBNA) has been shown to be an effective tool in the evaluation of malignant and benign enlarged mediastinal and hilar lymph nodes [1-4] and has become the procedure of choice [5-7].

Several studies have shown that EBUS-TBNA requires more time and greater sedation than conventional TBNA (without ultrasound) [3, 8]. Initially, general anesthesia (GA) or deep sedation was used, although moderate seda- 
tion (MS) was later considered sufficient $[9,10]$, with some debate [11-14]. Casal et al. [13] showed no differences in patient tolerance or major complications associated with GA or MS; GA may therefore be a safe alternative in cases of comorbidities or intolerance to MS.

Improvement in the learning curve of pulmonologists has reduced the examination time, and GA is currently being replaced by MS $[15,16]$, although GA or deep sedation are both accepted in the case of inexperienced pulmonologists or for complete lymph node staging [9].

Two objectives must be achieved in sedation during EBUS: tolerance of the examination with the least possible movement and coughing, while, at the same time, preventing respiratory depression, as airway patency is not maintained by devices such as the endotracheal tube or laryngeal mask airway [17]. Mild-to-moderate sedation with benzodiazepines and opioids $[18,19]$ is the type of sedation commonly used by pulmonologists, whereas moderate-to-deep sedation with propofol, alone or in association with opioids $[11,20,21]$, requires the presence of personnel trained to use these drugs, according to current guidelines [22-25]. Anesthesia protocols vary between hospitals and countries, and no protocol is applied uniformly.

In our hospital, sedation is carried out using propofol and remifentanil in continuous infusion due to their pharmacokinetic and pharmacodynamic synergies and because these are the two drugs that are being used lately during endoscopy with a high level of satisfaction [17, 26]. Propofol has a narrow therapeutic window, and the addition of an opiate to reduce cough and the dose of propofol may increase the risk of respiratory depression. Appropriate monitoring to prevent the risks of deep sedation is therefore required [27].

Clinical monitoring of sedation using the modified observer's assessment of alertness/sedation (MOAA/S) scale [28] has the disadvantage of requiring frequent stimulation for evaluation as well as its subjectivity. Monitoring of the depth of anesthesia using the bispectral index (BIS) $[29,30]$ allows us to objectively evaluate the level of sedation achieved and prevent the adverse events of overdose [31, 32]. The BIS monitor has been found to be useful for dosing drugs and reducing recovery time in patients under sedation in diagnostic and therapeutic procedures [33-35]. In fiberoptic bronchoscopy, it has been estimated that BIS levels for appropriate sedation are between 65 and 85 [36-39], and its ability to reduce the dose of sedatives has not been demonstrated [39]. EBUS is a longer procedure that requires a higher level of sedation, and the only information available on the use of
Table 1. Modified observer's assessment of alertness/sedation scale

\section{Responsiveness}

Agitated

Responds readily to name spoken in normal tone ('alert')

Lethargic response to name spoken in normal tone

Responds only after name is called loudly and/

or repeatedly

Responds only after mild prodding or shaking

Does not respond to mild prodding or shaking

Does not respond to deep stimulus

\section{Score}

6

5

4

2

1

0
BIS is limited to the control of the depth of sedation; its potential for reducing the dose of sedatives in a group of patients with respiratory disease, which may increase adverse events, has never been evaluated.

\section{Patients and Methods}

A randomized cohort study was performed in the Miguel Servet Hospital, Zaragoza, Spain, from May 20, 2014, to March 31, 2015 . Ninety patients with mediastinal lymph node involvement and/or lung or mediastinal lesions, in whom the EBUS-TBNA procedure was indicated, were studied to compare sedation between two groups: one group used BIS evaluation (BIS group) and the other used the MOAA/S [28] clinical scale (non-BIS group) (table 1). Patients with diseases or drug use with a potential effect on the central nervous system were excluded. The drug doses required, adverse events at different times, tolerance, and difficulty of the examination were evaluated in each group. The study was approved by the Ethics Committee of Hospital Universitario Miguel Servet, Zaragoza, Spain (approval number: 02/2014), and the patients provided written informed consent.

EBUS-TBNA was performed by experienced pulmonologists and pulmonary medicine residents, while sedation was performed by the same anesthesiologist. Patients were assigned to each group by means of simple random sampling. The BIS monitor was applied, and the values were recorded in both groups. However, the anesthetist was blinded to the BIS values in the non-BIS group. Hemodynamic variables and BIS values were recorded by a pulmonary nurse who was blinded to the patients' group allocation.

Demographic, comorbidity, and respiratory data were collected. While EBUS-TBNA was being performed, hemodynamic variables (arterial blood pressure and heart rate), respiratory variables (respiratory frequency, $\mathrm{SpO}_{2}$, and $\mathrm{EtCO}_{2}$ ), and BIS and MOAA/S values $[28,29]$ were recorded at each of the 9 different moments of the examination ( $\mathrm{t} 1$, baseline; $\mathrm{t} 2,1-5 \mathrm{~min} ; \mathrm{t} 3,5-10 \mathrm{~min}$; $\mathrm{t} 4$, insertion of fiberoptic bronchoscope; $t 5$, puncture; $t 6,30 \mathrm{~min}$; $\mathrm{t}$, 60 $\mathrm{min} ; \mathrm{t} 8$, removal of fiberoptic bronchoscope; $\mathrm{t} 9$, response to spoken orders). Total doses of the different drugs (propofol, remifentanil, and lidocaine) and different times were recorded: $T_{\mathrm{a}}$ : time between the start of sedation and loss of verbal contact in normal tones; $T_{b}$ : time between the end of sedation and verbal response in normal tones; $T_{c}$ : time between the end of sedation and BIS $>90$, 
and $\mathrm{T}_{\mathrm{d}}$ : duration of sedation. Finally, adverse events during the procedure and during the stay in the recovery unit, patient satisfaction, and difficulty experienced by the person performing the endoscopy were recorded. The cost of BIS is less than USD 15 per patient.

\section{Sedation Protocol}

- Propofol in continuous infusion by means of Alaris PK Carefusion Target Controlled Infusion pump; the infusion began with a plasma objective of $2 \mu \mathrm{g} / \mathrm{ml}$ until a site-effect concentration of $1 \mu \mathrm{g} / \mathrm{ml}$ was reached in the brain, increasing or reducing the plasma concentration by $0.3 \mu \mathrm{g} / \mathrm{ml}$ every minute to obtain a MOAA/S alertness/sedation score of 3 or 2 (MS [28]). Furthermore, in the BIS group, an effort was made to keep the BIS between 60 and 80 (MS [30]).

- Remifentanil in continuous infusion, at $0.04 \mu \mathrm{g} / \mathrm{kg} / \mathrm{min}$, except in patients over 70 years of age or with an American Society of Anesthesiologists (ASA) score of 3, in whom the infusion rate was reduced to $0.02 \mu \mathrm{g} / \mathrm{kg} / \mathrm{min}$. Previously, the pulmonologist (who, as the nurse, was unaware of which group the patient had been assigned to) anesthetized the vocal cords, trachea, and both main bronchi with $2 \%$ lidocaine, as per usual procedure. All patients received supplemental oxygen at 6 liters/min via a nasal cannula, with end-tidal $\mathrm{CO}_{2}$ monitoring.

\section{Statistical Analysis}

The results were reported using central tendency and dispersion for quantitative variables [mean, median, standard deviation (SD), and interquartile range (IQR)] and percentages for the qualitative variables. For the bivariate analysis, we used the $\chi^{2}$ or Fisher exact tests for the association between qualitative variables and the t test, ANOVA, Mann-Whitney test, and Kruskal-Wallis test for quantitative variables, depending on whether or not data distribution was normal, as evaluated by the Kolmogorov-Smirnov test. Correlation was analyzed using the Spearman rank correlation.

The tests were applied using the bilateral hypothesis, and the difference was considered to be statistically significant for values of $\mathrm{p}<0.5$. The confidence intervals included were 95\% (95\% CI). Data were analyzed using version 15 of the Statistical Package for Social Sciences (SPSS).

\section{Results}

Demographic variables and general reasons for requesting the procedure are shown in table 2 . The mean (SD) duration of the procedure was $75.5 \mathrm{~min}$ (16.2).

No statistically significant differences were observed in the number of adverse events by sex, age 65 years or more, smoking, obesity, or ASA group; differences were significantly greater depending on whether patients had suffered from prior obstructive sleep apnea (OSA; 3.61 adverse events in OSA patients compared to 2.90 in nonOSA patients; $p=0.049$ ) and a significant positive linear correlation was found between the number of adverse
Table 2. Demographic variables and general reasons for requesting the procedure, by sedation group

\begin{tabular}{|c|c|c|c|}
\hline & $\begin{array}{l}\text { Non-BISa } \\
\text { (MOAA/S) }\end{array}$ & BIS & $\begin{array}{l}\mathrm{p} \\
\text { value }\end{array}$ \\
\hline \multicolumn{4}{|l|}{ Sex } \\
\hline Male & $8(17.8)$ & $12(26.7)$ & \multirow[t]{2}{*}{0.310} \\
\hline Female & $37(82.2)$ & $33(73.3)$ & \\
\hline \multicolumn{4}{|l|}{ ASA } \\
\hline 1 & $2(4.4)$ & $2(4.4)$ & \multirow[t]{3}{*}{0.977} \\
\hline 2 & $24(53.3)$ & $25(55.6)$ & \\
\hline 3 & $19(42.2)$ & $18(40.0)$ & \\
\hline \multicolumn{4}{|l|}{$\mathrm{O}_{2}$} \\
\hline Yes & $1(2.2)$ & $1(2.2)$ & 1.000 \\
\hline \multicolumn{4}{|l|}{ Smoking } \\
\hline Yes & $11(24.4)$ & $17(37.8)$ & 0.172 \\
\hline \multicolumn{4}{|l|}{ Type of smoker } \\
\hline No & $08(17.8)$ & $07(15.6)$ & \multirow[t]{5}{*}{0.649} \\
\hline Ex-smoker $>3$ months & $23(51.1)$ & $18(40.0)$ & \\
\hline Ex-smoker $\leq 3$ months & $3(6.7)$ & $3(6.7)$ & \\
\hline Smoker $<10 \mathrm{c} /$ day & $4(8.9)$ & $4(8.9)$ & \\
\hline Smoker $\geq 10 \mathrm{c} /$ day & $7(15.6)$ & $13(28.9)$ & \\
\hline \multicolumn{4}{|l|}{ Cough } \\
\hline Yes & $28(62.2)$ & $25(55.6)$ & 0.520 \\
\hline \multicolumn{4}{|l|}{ OSA } \\
\hline Yes & $9(20)$ & $9(20)$ & 1.000 \\
\hline \multicolumn{4}{|c|}{ Previous fiberoptic bronchoscopy } \\
\hline Yes & $26(57.8)$ & $23(51.1)$ & 0.525 \\
\hline \multicolumn{4}{|l|}{ Indication } \\
\hline Lung cancer & $29(64.4)$ & $28(62.2)$ & 0.766 \\
\hline Metastasis & $9(20.0)$ & $10(22.2)$ & \\
\hline Sarcoidosis/lymphoma & $6(13.3)$ & $7(15.6)$ & \\
\hline Mediastinal mass & $1(2.2)$ & $0(0)$ & \\
\hline
\end{tabular}

Values are expressed as n (\%). $\mathrm{c}=$ Cigarettes. ${ }^{\mathrm{a}}$ Non-BIS: group guided by MOAA/S sedation score but with BIS recorded.

events during the procedure and the duration of the procedure $(\rho, 0.221 ; 95 \% \mathrm{CI}, 0.005-0.401 ; \mathrm{p}=0.045)$.

Moreover, no statistically significant differences were observed between the BIS and non-BIS groups in any demographic or comorbidity variable: sex, ASA score, smoking, domiciliary oxygen, OSA, previous fiberoptic bronchoscopy, indication of the procedure, age, weight, height, $\mathrm{BMI}, \mathrm{O}_{2}$ saturation, level of anxiety, forced expiratory pressure in $1 \mathrm{~s}$ and forced vital capacity (tables 3,4 ). Furthermore, the mean (SD) number of comorbidities in the non-BIS group was 3.64 (1.82) compared to 3.40 (1.70) in the BIS group; no statistically significant differences were found between the groups in any of the comorbidity variables taken into account $(\mathrm{p}=0.512)$.

Oxygen saturation and BIS values were of note among the hemodynamic and respiratory variables. Oxygen sat- 
Table 3. Sociodemographic, respiratory, and anxiety level variables by group

Table 4. Comorbidity variables by group

\begin{tabular}{lllllll}
\hline & \multicolumn{2}{l}{ Non-BIS (MOAA/S) } & & BIS & \multirow{2}{*}{ p value } \\
\cline { 2 - 3 } & median & IQR & & median & IQR & \\
\hline Age, years & 66.9 & $53.2-75.4$ & & 65.7 & $56.0-72.7$ & 0.707 \\
BMI & 26.8 & $23.8-30.2$ & & 26.3 & $22.0-30.4$ & 0.450 \\
SaO2, \% & 96 & $95.0-97.0$ & & 95 & $94.0-97.0$ & 0.182 \\
FEV,$\%$ & 86 & $72.0-96.0$ & & 83 & $67.5-98.0$ & 0.680 \\
Forced vital capacity, \% & 91 & $82.0-104.8$ & 91 & $82.0-114.0$ & 0.836 \\
Basal anxiety level (0-10) & 4 & $3.0-5.0$ & & 5 & $3.0-7.0$ & 0.087 \\
\hline
\end{tabular}

FEV1 = Forced expiratory pressure in $1 \mathrm{~s}$.

\begin{tabular}{|c|c|c|c|c|c|}
\hline & \multicolumn{2}{|c|}{ Non-BIS (MOAA/S) } & \multicolumn{2}{|c|}{ BIS } & \multirow[t]{2}{*}{$\mathrm{p}$ value } \\
\hline & $\mathrm{n}$ & $\%$ & $\mathrm{n}$ & $\%$ & \\
\hline Hypertension & 25 & 55.6 & 17 & 37.8 & 0.091 \\
\hline Atrial fibrillation & 3 & 6.7 & 5 & 11.1 & 0.714 \\
\hline Cerebrovascular accident & 3 & 6.7 & 5 & 11.1 & 0.714 \\
\hline COPD & 15 & 33.3 & 17 & 37.8 & 0.660 \\
\hline OSA & 9 & 20.0 & 9 & 20.0 & 1.000 \\
\hline Neoplasm & 37 & 82.2 & 34 & 75.6 & 0.438 \\
\hline Diabetes & 12 & 26.7 & 12 & 26.7 & 1.000 \\
\hline Dyslipidemia & 17 & 37.8 & 16 & 35.6 & 0.764 \\
\hline Obesity $(\mathrm{BMI} \geq 30)$ & 1 & 2.2 & 2 & 4.4 & 1.000 \\
\hline Ischemic heart disease & 6 & 13.3 & 5 & 11.1 & 0.748 \\
\hline Renal failure & 3 & 6.7 & 2 & 4.4 & 1.000 \\
\hline Heart failure & 0 & 0.0 & 1 & 2.2 & 1.000 \\
\hline Anxiety & 2 & 4.4 & 2 & 4.4 & 1.000 \\
\hline Other diseases & 14 & 31.1 & 19 & 42.2 & 0.274 \\
\hline
\end{tabular}

COPD $=$ Chronic obstructive pulmonary disease

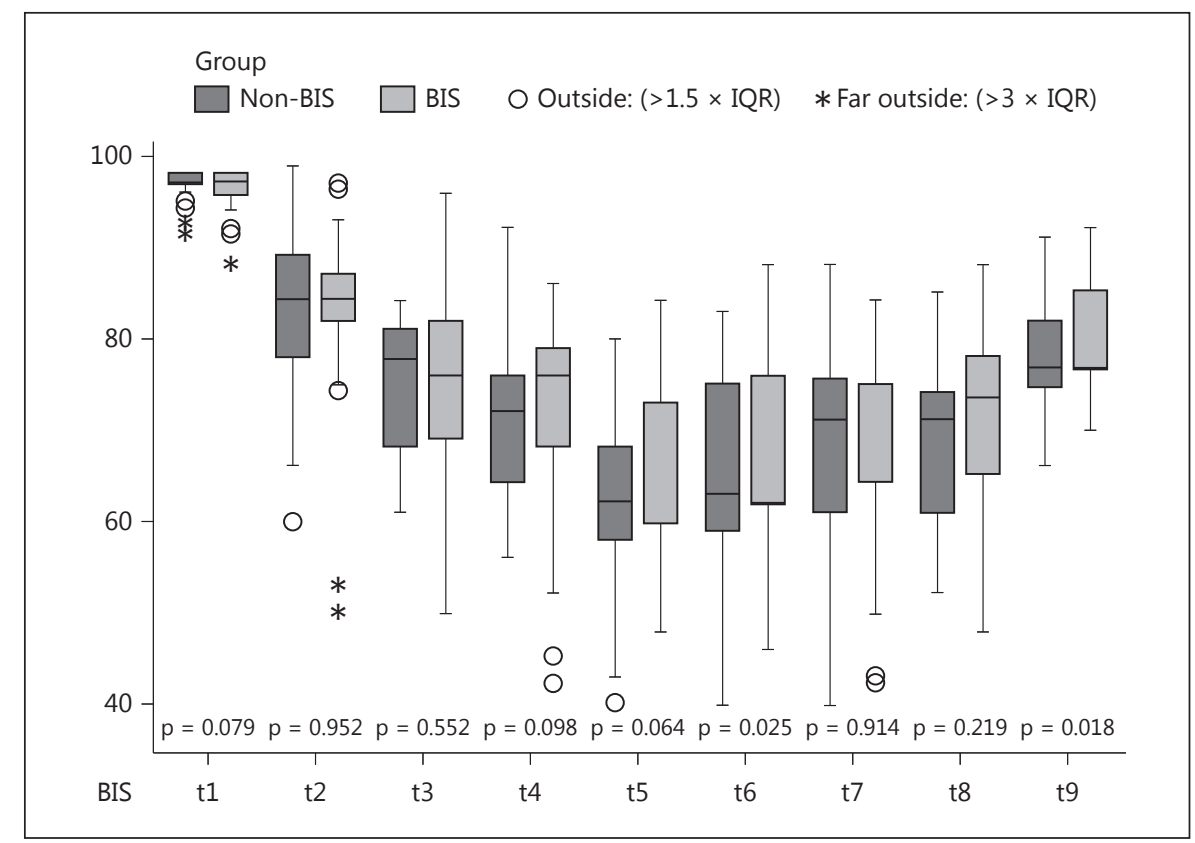


Fig. 2. Oxygen saturation values $\left(\mathrm{SaO}_{2}\right)$ at the 9 times during the procedure, by sedation group (non-BIS and BIS).

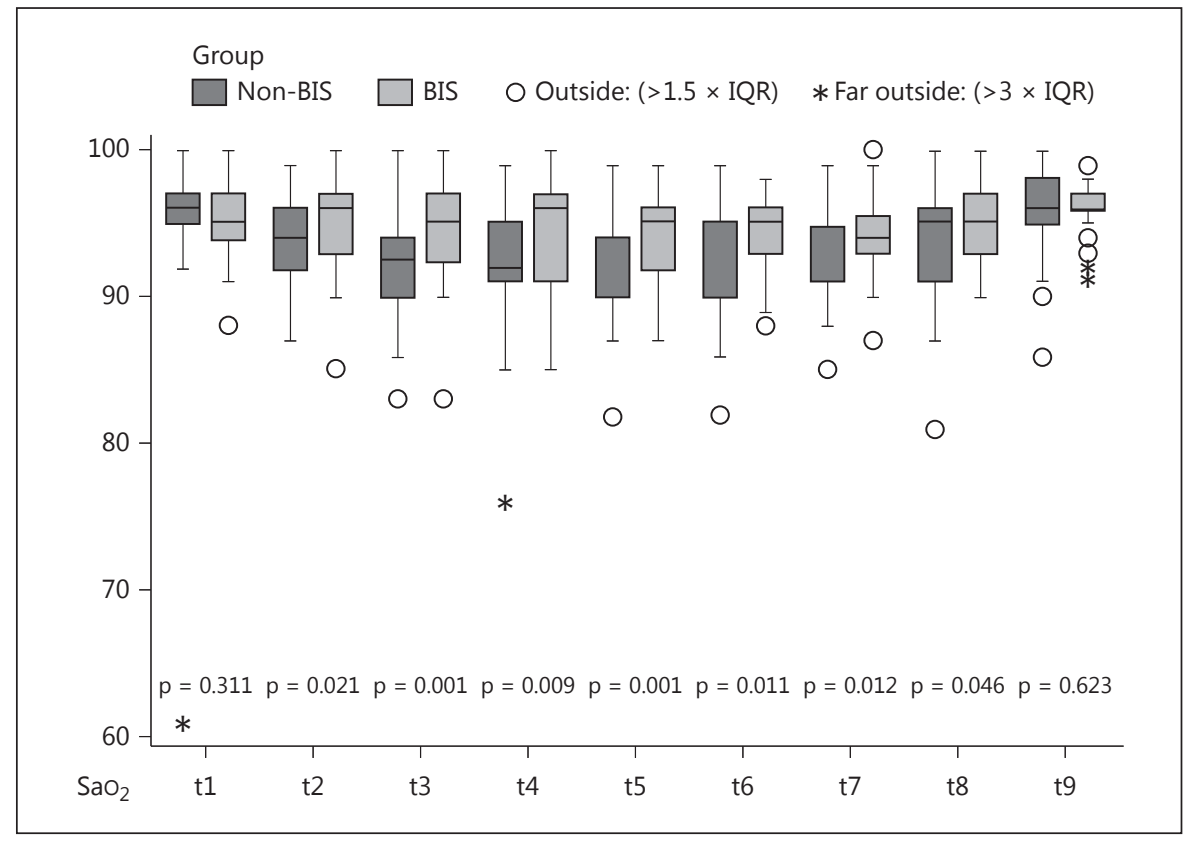

uration was significantly lower at all times (except at t1 and t9) in the non-BIS group, whereas the values in the BIS group were significantly higher at $\mathrm{t} 6$ and $\mathrm{t} 9$ in the BIS groups (fig. 1,2). Figure 3 shows the percentage of patients within the target range (MOAA/S 2-3), recorded at $\mathrm{t} 3-\mathrm{t} 7$ of the procedure. We did not include $\mathrm{t} 1, \mathrm{t} 2$, $\mathrm{t} 8$, and t9 because they are related with the induction and end of sedation.

Except for the time to loss of verbal contact, all times were greater in the non-BIS group; the difference in the time between the end of sedation and awakening (verbal response in a normal tone) was statistically significant $(\mathrm{p}=0.033)$. The mean doses of total propofol and propofol in $\mathrm{mg} / \mathrm{kg}$ were significantly higher in the non-BIS group, and the doses of lidocaine in $\mathrm{mg} / \mathrm{kg}$ were significantly higher in the BIS group (table 5).

The non-BIS group presented more adverse events [total, 164; mean (SD), 3.64 (1.20)] than the BIS group [total, 102; mean (SD), $2.27(0.86)$ ]; $(\mathrm{p}<0.001)$ (table 6). Twenty-seven (60\%) patients in the non-BIS group and 7 $(15.6 \%)$ in the BIS group required measures to counteract desaturation $(\mathrm{p}<0.001)$. Thus, the number of maneuvers carried out was higher in the first group [total, 54; mean (SD) per patient, $1.20(1.27)]$ than in the second group [total, 18; mean (SD) per patient, $0.40(0.96)$ ] $(\mathrm{p}<0.001)$.

Most patients (91.1\% in the non-BIS group and $84.4 \%$ in the BIS group) presented at least one cough attack (table 6). We found no significant differences in the number of cough attacks between the 2 groups [median (IQR), 4.0

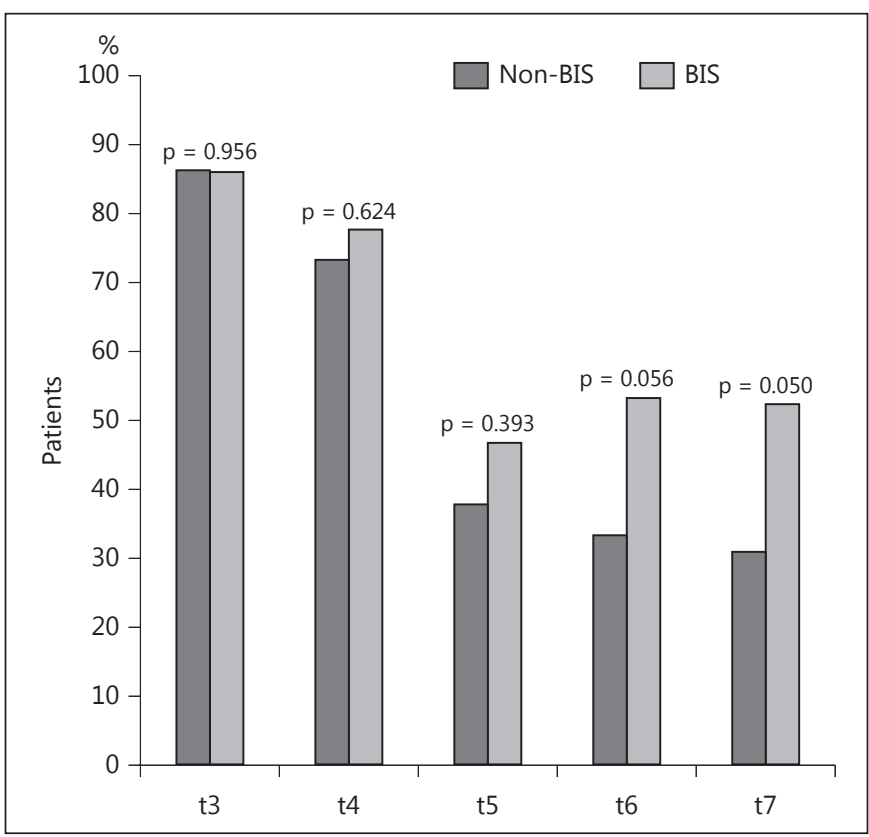

Fig. 3. Percent of patients within the target range (MOAA/S 2-3) at 5 times during the procedure ( $\mathrm{t} 3-\mathrm{t} 7)$, by sedation group (nonBIS and BIS).

(2.0-6.5) in the non-BIS group and $3.0(1.0-5.0)$ in the BIS group; $\mathrm{p}=0.115$ ]. Figure 4 shows patients with cough at each time in the procedure.

The mean (SD) number of adverse events in the postanesthesia care unit (PACU) in patients in the non-BIS 
Table 5. Statistics of the different procedure times and total and by-weight amounts of the drugs used during the procedure, by sedation group

\begin{tabular}{|c|c|c|c|c|c|}
\hline & Mean & Median & SD & IQR & $\mathrm{p}$ value \\
\hline \multicolumn{6}{|l|}{$\mathrm{T}_{\mathrm{a}}, \min$} \\
\hline Non-BIS & 5.1 & 5 & 2.5 & $3.0-7.0$ & \multirow[t]{2}{*}{0.407} \\
\hline BIS & 5.6 & 5 & 2.5 & $3.5-7.0$ & \\
\hline \multicolumn{6}{|l|}{$\mathrm{T}_{\mathrm{b}}, \min$} \\
\hline Non-BIS & 4.4 & 4 & 1.9 & $3.0-6.0$ & \multirow[t]{2}{*}{0.033} \\
\hline BIS & 3.6 & 3 & 1.9 & $2.0-5.0$ & \\
\hline \multicolumn{6}{|l|}{$\mathrm{T}_{\mathrm{c}}, \min$} \\
\hline Non-BIS & 8.4 & 8 & 3.3 & $6.5-11.5$ & \multirow[t]{2}{*}{0.299} \\
\hline BIS & 7.6 & 7 & 3.0 & $5.0-10.0$ & \\
\hline \multicolumn{6}{|l|}{$\mathrm{T}_{\mathrm{d}}, \min$} \\
\hline Non-BIS & 77.4 & 74 & 18.7 & $61.5-91.5$ & \multirow[t]{2}{*}{0.275} \\
\hline BIS & 73.6 & 74 & 13.5 & $62.0-86.5$ & \\
\hline \multicolumn{6}{|l|}{ Propofol, mg } \\
\hline Non-BIS & 534.4 & 475 & 206.5 & $367.0-679.0$ & \multirow[t]{2}{*}{0.001} \\
\hline BIS & 397.8 & 395 & 98.6 & $335.0-468.5$ & \\
\hline \multicolumn{6}{|c|}{ Propofol, mg/kg } \\
\hline Non-BIS & 6.8 & 6.4 & 2.2 & $4.9-8.5$ & \multirow[t]{2}{*}{0.006} \\
\hline BIS & 5.6 & 5.3 & 1.8 & $4.4-6.4$ & \\
\hline \multicolumn{6}{|c|}{ Remifentanil, $\mu \mathrm{g}$} \\
\hline Non-BIS & 180.5 & 147 & 86.6 & $117.5-220.5$ & \multirow[t]{2}{*}{0.347} \\
\hline BIS & 167.7 & 141 & 91.7 & $110.0-205.0$ & \\
\hline \multicolumn{6}{|c|}{ Remifentanil, $\mu \mathrm{g} / \mathrm{kg}$} \\
\hline Non-BIS & 2.31 & 2.12 & 0.97 & $1.54-2.83$ & \multirow[t]{2}{*}{0.672} \\
\hline BIS & 2.29 & 1.84 & 1.09 & $1.44-3.09$ & \\
\hline \multicolumn{6}{|l|}{ Lidocaine, $\mathrm{ml}$} \\
\hline Non-BIS & 33.9 & 32.0 & 13.1 & $25.0-45.0$ & \multirow[t]{2}{*}{0.171} \\
\hline BIS & 37.5 & 40.0 & 11.1 & $30.0-45.0$ & \\
\hline \multicolumn{6}{|c|}{ Lidocaine, $\mathrm{mg} / \mathrm{kg}^{\mathrm{a}}$} \\
\hline Non-BIS & 8.87 & 8.82 & 3.39 & $6.67-11.16$ & \multirow[t]{2}{*}{0.021} \\
\hline BIS & 10.76 & 10.00 & 4.18 & $8.00-14.26$ & \\
\hline
\end{tabular}

${ }^{a}$ Normal variables. group was $1.42(0.99)$, which was higher than that in the BIS group [mean (SD), $1.02(0.78) ; \mathrm{p}=0.036$ ]. Only 2 patients from each group had any memory of the procedure. Tolerance of the procedure was better $(p=0.03)$ in the BIS group, whereas the difficulty experienced by the endoscopist was similar in both groups (table 7).

\section{Discussion}

This prospective cohort study compares sedation during ultrasound-guided bronchoscopy with propofol and remifentanil using BIS versus the MOAA/S clinical scale and shows that BIS not only controls the depth of sedation but also reduces the adverse events associated with sedatives, particularly hypotension, desaturation, and bradypnea, by making it possible to reduce the dose of these drugs. It also improves tolerance and reduces the waking time.

BIS Reduces Adverse Events in EBUS Sedation
Table 6. Comparison of adverse events during the procedure between the sedation groups

\begin{tabular}{|c|c|c|c|c|}
\hline \multirow[t]{2}{*}{ Adverse events } & \multicolumn{2}{|c|}{ Non-BIS } & BIS & \multirow[t]{2}{*}{$\mathrm{p}$ value } \\
\hline & $\mathrm{n}$ & $\%$ & $\mathrm{n} \%$ & \\
\hline Cough attacks & 41 & 91.1 & 3884.4 & 0.334 \\
\hline Oral aspiration & 9 & 20.0 & 511.1 & 0.245 \\
\hline Desaturation & 27 & 60.0 & 715.6 & $<0.001$ \\
\hline Bradypnea & 18 & 40.0 & 511.1 & 0.002 \\
\hline $\mathrm{ETCO}_{2} \geq 50$ & 9 & 20.0 & 36.7 & 0.063 \\
\hline Hypotension & 45 & 100.0 & 2760.0 & $<0.001$ \\
\hline Hypertension & 2 & 4.4 & 12.2 & 0.557 \\
\hline Bradycardia & 13 & 28.9 & 1431.1 & 0.818 \\
\hline Tachycardia & 0 & 0.0 & 24.4 & 0.494 \\
\hline
\end{tabular}

Bradypnea: breathing rate $<8$ breaths/min. Desaturation: oxygen saturation $<90 \%$ for more than $1 \mathrm{~min}$. Arterial hypotension: $<20 \%$ baseline mean blood pressure. Arterial hypotension: $>20 \%$ baseline mean blood pressure. Bradycardia: fall of $15 \mathrm{bpm}$ below baseline. Tachycardia: rise of $30 \mathrm{bpm}$ above baseline. 
Fig. 4. Percent of patients with cough attacks in both groups at 9 times during the procedure. n.a. $=$ Not applicable.

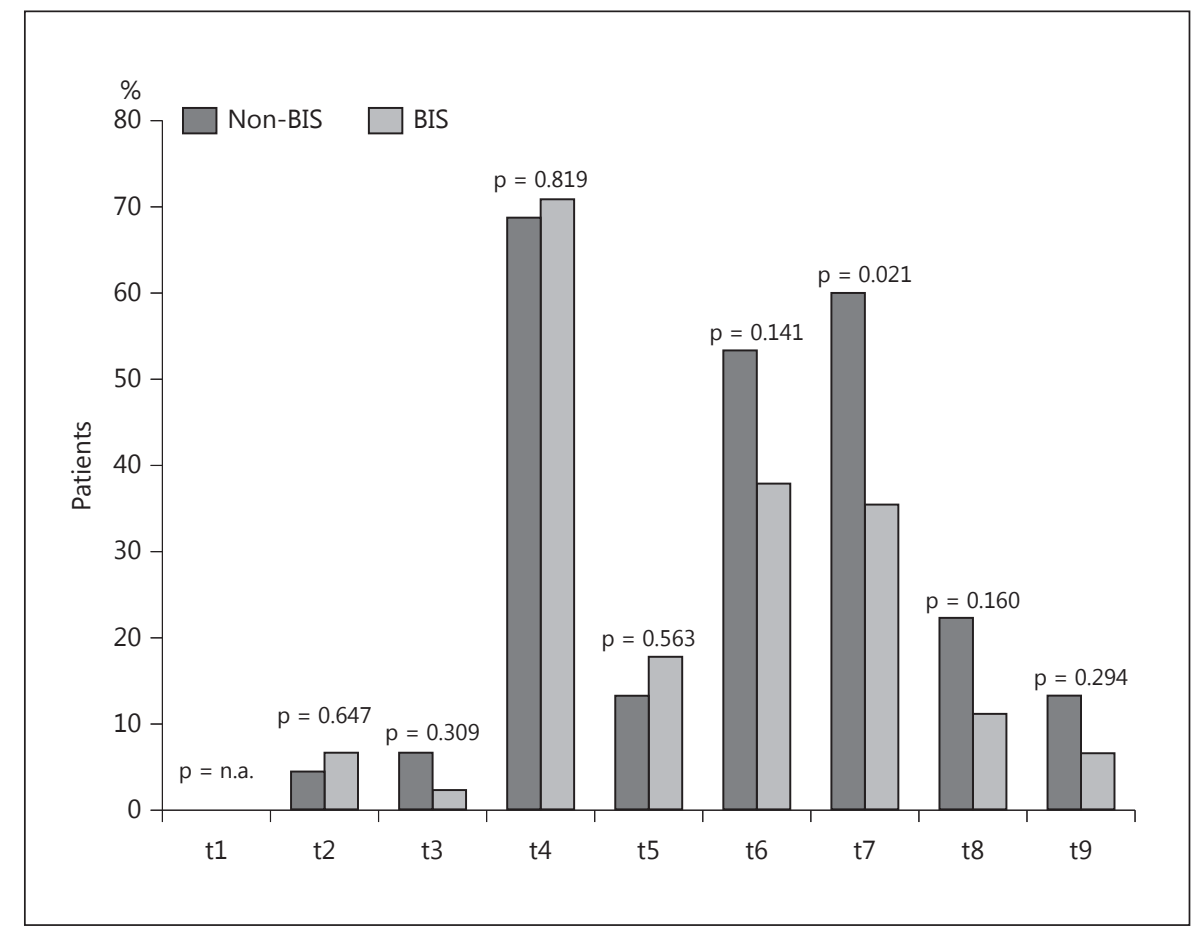

Table 7. Adverse events in the postanesthesia recovery unit, tolerance, and difficulty perceived by the endoscopist

\begin{tabular}{|c|c|c|c|c|c|}
\hline & \multicolumn{2}{|l|}{ Non-BIS } & \multicolumn{2}{|l|}{ BIS } & \multirow[t]{2}{*}{$\mathrm{p}$ value } \\
\hline & $\mathrm{n}$, median & $\%, I Q R$ & $\mathrm{n}$, median & $\%$, IQR & \\
\hline Sore throat in PARU ${ }^{\mathrm{a}, \mathrm{b}}$ & 18 & 40.0 & 13 & 28.9 & 0.267 \\
\hline Chest pain in PARU ${ }^{\mathrm{b}}$ & 8 & 17.8 & 1 & 2.2 & 0.030 \\
\hline Cough in PARU ${ }^{b}$ & 30 & 66.7 & 26 & 57.8 & 0.384 \\
\hline Dyspnea in PARU ${ }^{b}$ & 5 & 11.1 & 3 & 6.7 & 0.714 \\
\hline Memory & 2 & 4.4 & 2 & 4.4 & 1.000 \\
\hline Would not repeat ${ }^{b}$ & 1 & 2.2 & 1 & 2.2 & 1.000 \\
\hline Not discharged ${ }^{\mathrm{b}}$ & 0 & 0.0 & 0 & 0.0 & - \\
\hline Patient tolerance ${ }^{c}$ & 9.0 & $8.0-10.0$ & 10.0 & $9.0-10.0$ & 0.003 \\
\hline Difficulty perceived by endoscopist ${ }^{\mathrm{d}}$ & 9.0 & $7.0-9.5$ & 9.0 & $8.0-9.0$ & 0.466 \\
\hline
\end{tabular}

${ }^{\mathrm{a}}$ PARU $=$ Postanesthesia recovery unit. ${ }^{\mathrm{b}}$ Categorical variables (yes/no). ${ }^{\mathrm{c}}$ Patient tolerance: ordinal variable from 0 (uncomfortable) to 10 (comfortable). ${ }^{\mathrm{d}}$ Difficulty perceived by the endoscopist: ordinal variable from 0 (very difficult) to 10 (very easy).

As in previous studies on digestive endoscopy [40], we found a positive linear correlation between the number of adverse events and the duration of the procedure (mean $75.5 \mathrm{~min}$ ). These adverse events with the longer duration are considered to be linked to the higher doses of sedatives, and it has been suggested that doses of $\geq 100$ $\mathrm{mg}$ of propofol lead to more adverse events. Other authors found no relationship between adverse events and the duration of EBUS-TBNA $[10,21]$, but the mean duration of their procedures was shorter. Our longer mean duration may have been due to the learning curve of the residents and the systematic triple puncture of all lymph node stations punctured, which included lymph nodes not captured on the positron emission tomography scan. As in our study, other authors have found no correlation between adverse events and advanced age or higher ASA scores $[10,13,41]$, although OSA did correlate with adverse events. Our results, which made it possible to reduce the dose of drugs in the BIS group, suggest that the lower incidence of adverse events in this group is linked 
to the lower dose of the drugs used. No previous studies have evaluated monitoring with BIS and its effect on the dose of propofol during EBUS. In our study, patients monitored using BIS required significantly lower total mean doses of propofol throughout the procedure, and it was also subsequently shown that patients monitored without BIS reached deeper levels of sedation, related to the larger doses of drugs, and required more time to wake up.

Overall desaturation observed in our patients was $37.8 \%$, with statistically significant differences between the groups (non-BIS, $60.0 \%$; BIS, $15.6 \%$; $\mathrm{p}=0.001$ ); sedation was not as deep in the BIS group (levels of 70-75 compared to 60-70 in the non-BIS group), and this was associated with less desaturation. The incidence in the BIS group was far lower than that reported by Jensen [20] (50\%), who used only propofol, though at higher doses, and by Vila [17] (47.8\%), who also used remifentanil. The incidence in the Cases Viedma et al. [21] series, with a combination of propofol and remifentanil, was similar to that in our BIS group (12.9\%), although this multicenter study did not specify the dose or level of sedation. This lower hypoxemia is attributable to less severe respiratory depression, with less compromising of the airway, due to the more limited use of propofol and, therefore, less severe relaxation of the pharyngeal muscles, and to the lower incidence of bradypnea in the BIS group. Other potential causes of hypoxemia, common to both groups, include obstruction of the tracheal lumen by the endoscope and cough. However, no intubation was performed, and no procedure was suspended due to desaturation, as these events were considered to be transitory because the patients improved as a result of the actions carried out, particularly elevation of the jaw, continuous positive airway pressure (CPAP), and placement of a Valentín Madrid oropharyngeal cannula $\left(\mathrm{VAMA}^{\circledR}\right)$. In most cases, conventional fiberoptic bronchoscopy was performed before the EBUS procedure, and we were able to manage possible desaturation events. CPAP or VAMA were applied before starting the EBUS procedure. In other cases, we rarely had to interrupt the procedure to apply CPAP or to place VAMA. We used CPAP and VAMA in our OSA patients, but GA may be a safer alternative in these patients. The greater use of lidocaine in the BIS group may be related to the lower doses of propofol in these patients, but our dose of lidocaine was, in both groups, above the maximum recommended $(8.3 \mathrm{mg} / \mathrm{kg})$. However, lidocaine absorption can be unpredictable due to frequent aspirations during the procedure, and we found no objective toxic effects of lidocaine. Nevertheless, the patients from both groups showed a high cough rate but a limited number of cough attacks, and we should probably have used a lower dose of lidocaine. However, it does not seem logical to increase propofol or remifentanil infusion due to cough alone because of a higher risk of major side effects such as hypotension, bradypnea, and desaturation.

The overall incidence of hypotension in our study was $80 \%$, although it was much lower in the BIS group (non$\mathrm{BIS}, 100 \%$; BIS, $60 \% ; \mathrm{p}=0.001$ ). This percentage is greater than in other studies, as our criterion for hypotension was a reduction of $20 \%$ from the baseline mean blood pressure, not a $30 \%$ drop [21]. Hypotension events resolved spontaneously after adjusting sedative doses in accordance with sedation protocol (BIS and clinical in non$\mathrm{BIS}$, respectively) or increasing fluid therapy. We did not need to use vasoactive drugs in any case. Propofol has been linked to hypotension in moderate and deep sedation [42]. The lower incidence of hypotension in the BIS group, associated with the use of less propofol, is another advantage of this monitoring. Our awakening time (verbal response in normal tones, or $\mathrm{Tb}$ ) was 4.4 min (nonBIS) and 3.6 min (BIS), shorter than in another study [38] that assessed 'time for orientation'.

In the PACU, the mean number of different adverse events per patient in the non-BIS group was significantly greater than in the BIS group; the most frequent events were cough and sore throat. Of our patients, $98 \%$ did not remember the procedure, and there were no differences between the groups. It has been established that, with BIS scores of between 70 and $75,95 \%$ of the patients have no memory of the procedure $[29,30]$. Our patients in the BIS group only presented a significantly higher BIS score at some of the recorded times (30 min and on responding to verbal orders), but there were no differences between the two groups in the overall scores, which were sufficient to generate amnesia. Although the overall satisfaction (patients who would repeat the procedure) was very high $(98 \%)$, a rate similar to that found by other authors $[10,19]$ and with no differences between the groups, the tolerance assessed using a numerical scale was higher in the BIS group ( $\mathrm{p}=0.003)$, which led to a higher level of comfort in these patients, probably in relationship to a shorter recovery time, and to a lower degree of dizziness.

The main limitations of this study include the limited number of patients, as the study was carried out in a single hospital. However, this meant that it was possible for the same team (the same anesthetist, the same pulmon- 
ologists, and the same nurses) to carry out all procedures, allowing for little variability.

In conclusion, our study shows the utility of BIS in EBUS, not only for monitoring sedation but also for reducing the dose of drugs and thus their adverse events, at a very low cost. Studies with a larger number of patients may help with the systematic implementation of this form of monitoring.

\section{Acknowledgement}

The authors thank the nursing staff of the section of the pulmonary endoscopy of Miguel Servet Hospital.

\section{Financial Disclosure and Conflicts of Interest}

The authors declare that they have no conflicts of interest to disclose.

\section{References}

1 Krasnik M, Vilmann P, Larsen SS, Jacobsen GK: Preliminary experience with a new method of endoscopic transbronchial real time ultrasound guided biopsy for diagnosis of mediastinal and hiliar lesions. Thorax 2003;58: 1083-1086.

- Anantham D, Koh MS, Ernst A: Endobroncial ultrasound. Respir Med 2009;103:1406-1414.

-3 Temblay A, Slather DR, Maceachen P, Khalil M, Field SK: A randomized controlled trial of standard vs endobronchial ultrasonnography-guided transbronchial needle aspiration in patiens with suspected sarcoidosis. Chest 2009;136:340-346.

4 Morikawa K, Kurimoto N, Inoue T, Mineshita M, Miyazawa T: Histogram-based quantitative evaluation of endobronchial ultrasonography images of peripheral pulmonary lesion. Respiration 2015;89:148-154.

5 Yasufuku K: Current clinical applications of endobronchial ultrasound. Expert Rev Respir Med 2010;4:491-498.

6 Colt HG, Davoudi M, Murgu S: Scientific evidence and principles for the use of endobronchial ultrasound and transbronchial needle aspiration. Expert Rev Med Devices 2011;8: 493-513.

7 Unroe MA, Shofer SL, Wahidi MM: Training for endobronchial ultrasound : methods for proper training in new bronchoscopic techniques. Curr Opin Pulm Med 2010;16:295300.

8 Plat G, Pierard P, Haller A, Hutsebaut J, Faber J, Dusart M, Eisendrath P, Sculier JP, Ninane $\mathrm{V}$ : Endobronchial ultrasound and positrón emission tomography positive mediastinal lymph nodes. Eur Respir J 2006;27:276-281.

-9 Kennedy MP, Shweihat Y, Sarkiss M, Eapen GA: Complete mediastinal and hiliar lymph node staging of primary lung cancer by endobronchial ultrasound: moderate sedation or general anesthesia? Chest 2008;134:3501351.

-10 Sazak H, Tunç M, Alagöz A, Pehlivanoğlu P, Demirci NY, Alıci İO, Yılmaz A: Assessment of perianesthetic data in subjects undergoing EBUS-TBNA. Respir Care 2015;60:567-576.

-11 Yarmus LB, Akulian JA, Gilbert C: Comparison of moderate versus deep sedation for endobronchial ultrasound transbronchial nee- dle aspiration. Ann Am Thorac Soc 2013;10: 121-126.

12 Hsia DW, Jensen KW, Currant-Everett D, Musani AI: Diagnosis of lung nodules with peripheral/radial endobronchial ultrasound guided transbronchial biopsy. J Bronchol Interv Pulmonol 2012;19:5-11.

13 Casal RF, Lazarus DR, Nogueras-González G, Perusich S, Green LK, Ost DE, Sarkiss M, Jimenez CA, Eapen GA, Morice RC, Cornwell L, Austria S, Sharafkanneh A, Rumbaut RE, Grosu H, Kheradmand F: Randomized trial endobronchial ultrasound-guided transbronchial needle aspiration under general anesthesia versus moderate sedation. Am J Respir Crit Care Med 2015;191:796-803.

14 Van der Heijden EH, Casal RF, Trisolini R, Steinfort DP, Hwangbo B, Nakajima T, Guldhammer-Skov B, Rossi G, Ferretti M, Herth FF, Yung R, Krasnik M; World Association for Bronchology and Interventional Pulmonology, Task Force on Specimen Guidelines: Guideline for the acquisition and preparation of conventional and endobronchial ultrasound-guided transbronchial needle aspiration specimens for the diagnosis and molecular testing of patients with known or suspected lung cancer. Respiration 2014;88:500-517.

15 Monsó E, Andreo F, Rosell A, Cuellar P, Castellà E, Llatjós M: Usefulness of endobronchial ultrasonography with real-time needle aspiration for lung cancer staging. Med Clin 2007;128:481-485.

16 Du Rand IA, Barber PV, Goldring J, Lewis RA, Mandal S, Munavvar M, Rintoul RC, Shah PL, Singh S, Slade MG, Woolley A: British Thoracic Society guideline for advanced diagnostic and therapeutic flexible bronchoscopy in adults. Thorax 2011;66(suppl 3):iii1iii21.

17 Vila E, Mases A, Vela E, Moltó L, Molto L, Sanchez-Font A, Curull V, Gallart L: Sedación con propofol y remifentanilo para la ultrasonografía endobronquial con punción-aspiración en tiempo real. Rev Colom Anestesiol 2013;41:120-126

18 Jeyabalan A, Medford AR: Endobronchial ultrasound-guided transbronchial needle aspiration: patient satisfaction under light con- scious sedation. Respiration 2014;88:244250.

19 Steinfort DP, Irving LB: Patient satisfaction during EBUS-TBNA performed under conscious sedation. Respir Care 2010;55:702706.

20 Jensen JT, Banning AM, Clementsen P, Hammering A, Hornslet T, Vilmann P: Nurse administered propofol sedation for pulmonary endoscopies requires a specific protocol. Dan Med J 2012;59:A4467.

21 Cases Viedma E, Andreo García F, Flandes Aldeyturriaga J, Reig Mezquida JP, Briones Gómez A, Vila Caral P, Fernández-Navamuel Basozabal I, Centeno Clemente CÁ, Campo Campo F, Sánchez Martínez E, Sanchis Moret F, Manjón Pérez J, Sánchez Yepes M: Tolerance and safety of 5 models of sedation during endobronchial ultrasound (in English, Spanish). Arch Bronconeumol 2016;52:5-11.

22 Du Rand IA, Blaikley J, Booton R, Chaudhuri N, Gupta V, Khalid S, Mandal S, Martin J, Mills J, Navani N, Rahman NM, Wrightson JM, Munavvar M: British Thoracic Society guideline for diagnostic flexible bronchoscopy in adults: accreditd by NICE. Thorax 2013; 68(suppl1):i1-i44.

23 Wahidi MM, Jain P, Jantz M, Lee P, Mackensen GB, Barbour SY, Lamb C, Silvestri GA: American College of Chest Physicians Consensus Statement on the use of topical anesthesia, analgesia, and sedation during flexible bronchoscopy in adult patients. Chest 2011; 140:1342-1350.

24 American Society of Anesthesiologists Task Force on Sedation and Analgesia by Non-Anesthesiologists: Practice guidelines for sedation and analgesia by non-anesthesiologists. Anesthesiology 2002;96:1004-1017.

25 Knape JTA, Adriaensen $\mathrm{H}$, van Aken H, Blunnie WP, Carlsson C, Dupont MJ, Pasch T: Guidelines for sedation and/or analgesia by non-anesthesiology doctors. Eur J Anesthesiol 2007;24:563-567.

26 Yoon HI, Kim JH, Lee JM, Lee CT, Hwang JY, Nahm SF, Han S: Comparison of propofol and the combination of propofol and alfentanil during bronchoscopy: a randomized study. Acta Anaesthesiol Scand 2011;55:104109 
27 American Society of Anesthesiologists: Continuum of depth of sedation: definition of general anesthesia and levels of sedation/analgesia (approved by the ASA House of Delegates on October 13,1999, and last amended on October 15, 2014).

28 Chernick DA, Gillings D, Laine H, Hendler J, Silver JM, Davidson AB, Schwam EM, Siegel JL: Validity and reliability of the OAA/S study with iv midazolam. J Clin Psychofarmacol 1990;10:244.

29 Glass PS, Bloom M, Kearse L, Rosow C, Sebel P, Manberg P: Bispectral analysis measures sedation and memory effects of propofol, midazolam, isoflurane, and alfentanil in healthy volunteers. Anesthesiology 1997;86: 836-847.

30 Johansen JW: Update on bispectral index monitoring. Best Pract Res Clin Anaesthesiol 2006;20:81-99.

31 Liu J, Singh H, White PF: Electroencephalographic bispectral correlates with intraoperative recall and depth of propofol-induced sedation. Anesth Analg 1997;84:185-189.

32 Miner JR, Bachman A, Kosman L, Ross K: The utility of the bispectral index in procedural se- dation with propofol in the emergency department. Acad Emerg Med 2005;12:190196.

33 Wehrmann T, Groctkamp J, Stergiou N, Riphaus A, Kluge A, Lembcke B, Schultz A: Electroencephalogram monitoring facilitates sedation with propofol for routine ERCP: a randomized, controlled trial. Gastrointest Endosc 2002;56:817-824.

34 De Witt JM: Bispectral index monitoring for NAPS during upper endoscopic ultrasound: a prospective, randomized controlled trial. Dig Dis Sci 2008;53:2739-2745.

35 Punjasawadwong Y, Phongchiewboon A, Bunchungmongkol N: Bispectral index for improving anaesthetic delivery and postoperative recovery. Cochrane Database Syst Rev, 2014;17:CD003843.

36 Cases Viedma E, Pérez Pallarés J, Martínez García MA, López Reyes R, Sanchís Moret F, Sanchís Aldás JL: A randomised study of midazolam for sedation in flexible bronchoscopy. Arch Bronconeumol 2010;46:302-309.

- 37 Clark G, Licker M, Younossian AB, Soccal PM, Frey JG, Rochat T, Diaper J, Bridevaux PO, Tschopp JM: Titrated sedation with pro- pofol or midazolam for FB: a randomized trial. Eur Respir J 2009;34:1277-1283.

- 38 Lo Y, Lin TY, Fang YF, Wang TY, Chen HC, Chou CL, Chung FT, Kuo CH, Feng PH, Liu $\mathrm{CY}$, Kuo H: Feasibility of BIS-guided propofol infusion for flexible bronchoscopy sedation: a randomized controlled trial. PLoS One 2011;6:e27769.

-39 Fruchter O, Tirosh M, Carmi U, Rosengarten D, Kramer MR: Prospective randomized trial of BIS of sedation during flexible bronchoscopy. Respiration 2014;87:388-393.

40 Wehrmann T, Riphaus A: Sedation with propofol for interventional endoscopic procedures: a risk factor analysis. Scand J Gastroenterol 2008;43:368-374.

41 Eapen GA, Shah AM, Lei X, Jimenez CA, Morice RC, Yarmus L, Filner J, Ray C, Michaud G, Greenhill SR, Sarkiss M, Casal R, Rice D, Ost DE: Complications, consequences, and practice patterns of EBUS-TBNA: results of the AQuiRE registry. Chest 2013;143:1044-1053.

42 Ebert TJ: Sympathetic and hemodynamic effects of moderate and deep sedation with propofol in humans. Anesthesiology 2005;103: 20-24. 